\title{
In situ Analysis of the EUV Resist Pattern Formation during the Resist Dissolution Process
}

\author{
Julius Joseph Santillan and Toshiro Itani \\ EUVL Infrastructure Development Center, Inc. (EIDEC), \\ 16-1 Onogawa, Tsukuba, Ibaraki 305-8569, Japan \\ julius.santillan@eidec.co.jp
}

\begin{abstract}
The dissolution characteristics of EUV resist patterns as they are formed during the development process were analyzed using the HS-AFM. This in situ dissolution analysis method has been significantly optimized and can now be utilized for the analysis of $32 \mathrm{~nm}$ hp $\mathrm{L} / \mathrm{S}$ pattern developed with a standard concentration $(0.26 \mathrm{~N})$ of the tetramethyl ammonium hydroxide developer solution. Further investigations have shown that this method is presently limited to $\mathrm{hp} \mathrm{L} / \mathrm{S}$ patterns around $32 \mathrm{~nm} \mathrm{hp} \mathrm{L/S.} \mathrm{This} \mathrm{was} \mathrm{mainly} \mathrm{attributed} \mathrm{to} \mathrm{the}$ comparatively thick radius of curvature of presently available cantilevers. Furthermore, the results obtained here have shown that resists of good pattern LWR exhibit smooth patterns even during dissolution. On the other hand, resists of comparatively large LWR also show signs of such roughness even during the dissolution process. These results show the possibility of defining the formation mechanism of LWR during the development process.
\end{abstract}

Keywords: Resist dissolution, in situ, high speed atomic force microscopy, measurement limits, LWR

\section{Introduction}

With extreme ultraviolet (EUV) resist lithography targets for resolution, line width roughness (LWR), and sensitivity progressing to fine patterning beyond the $16 \mathrm{~nm}$ half-pitch (hp) lines and spaces $(\mathrm{L} / \mathrm{S})$, reconsideration of present resist formulations and related processes is necessary ${ }^{[1]}$. For resist material research, investigations on alternative resist formulations / platforms ${ }^{[2-4]}$ have shown potential results. For resist processes, alternative developers, rinse solutions, and post-processing techniques have been proposed ${ }^{[5-9]}$ in order to further improve the lithographic performance of the currently available resists. This shift to such new technologies leads to the need for further fundamental studies to better understand the resist materials and processes in order to achieve the stringent requirements.

Resist dissolution during the development process is one such area of research that has been thoroughly investigated ${ }^{[10-20]}$. The authors have proposed a direct approach using an in-liquid high-speed atomic force microscope (HS-AFM) for the in situ analysis of the resist dissolution behavior of actual resist patterns ${ }^{[21-25]}$. This allows a visual appreciation of the pattern formation on resist films, as it occurs and may provide new pointers for the further optimization of resist performance.

Earlier works were focused on isolated line patterns at diluted developer concentration. However, in recent months, significant technical advancements in cantilever technology and tool research and development have led to the stable analysis of the $\mathrm{hp} \mathrm{L/S}$ pattern with standard developer concentrations ${ }^{[26]}$. This paper, describes our investigation on the present capabilities and limitations of this methodology for application in the dissolution analysis of EUV resist patterns. This also focuses on the possible analysis applications that are currently being investigated. 


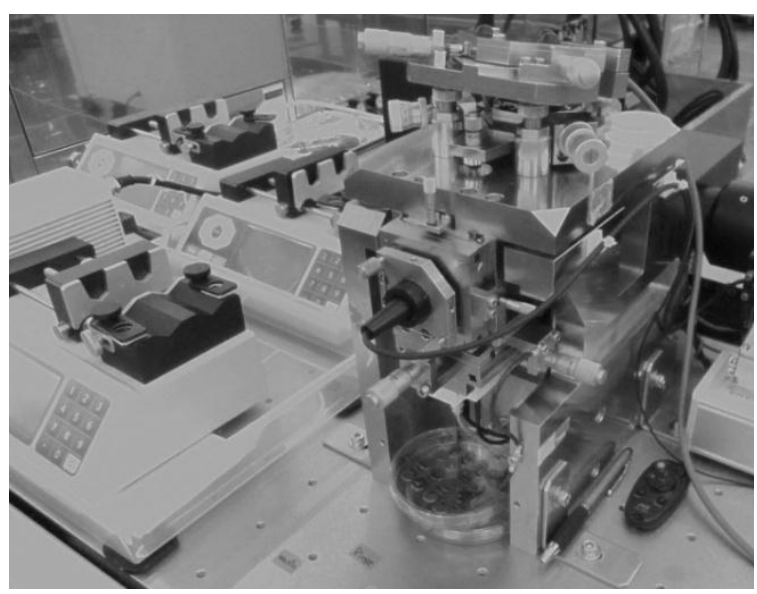

Fig. 1 The HS-AFM (Nano Explorer).

\section{Experimental conditions}

2.1 Materials and processing

These experiments were conducted using the EIDEC standard resist 1 (ESR1), a hybrid polyhydroxystyrene (PHS)-methacryl-based resist and Resist A, which is a litho-optimized hybrid (PHS-methacryl) resist. Both resists are utilized at a film thickness of $50 \mathrm{~nm}$. In addition, a hybrid PHS-methacryl model resist with $60 \mathrm{~nm}$ film thickness was utilized. Optimum post-application and post-exposure bake conditions were applied. These resists are chemical amplification and positive-tone type.

Silicon wafers with a diameter of $300 \mathrm{~mm}$ were coated with resist and were exposed to EUV using a small-field exposure tool (SFET; numerical aperture $=0.3$ ), which was linked to a coater / developer track system (Tokyo Electron Clean Track ACT12) in a chemically controlled environment. The pattern-exposed section of the wafer was then cleaved to obtain $2 \mathrm{~mm} \times 2 \mathrm{~mm}$ samples for the resist dissolution analysis using the HS-AFM system (Nano Explorer or NEX, Research Institute of Biomolecule Metrology Co., Ltd.). ${ }^{[26]}$ Figure 1 shows the HS-AFM inside the super clean room.

\subsection{Description of methodology}

The HS-AFM system is based on an early model reported previously ${ }^{[27]}$, but it has been extensively developed for application to in situ resist dissolution characterization.

Figure 2 shows the procedure utilized for the in situ resist dissolution analysis. Fig. 2(a) shows the following process: after the assembly and set up of the EUV-exposed resist sample and carbon nanofiber $(\mathrm{CNF})$ cantilever, a pre-determined

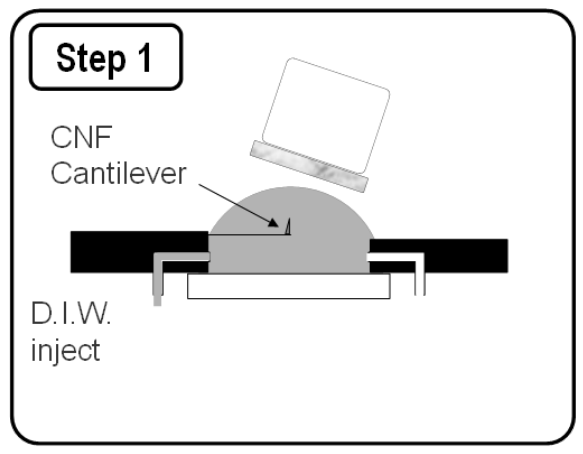

(a)

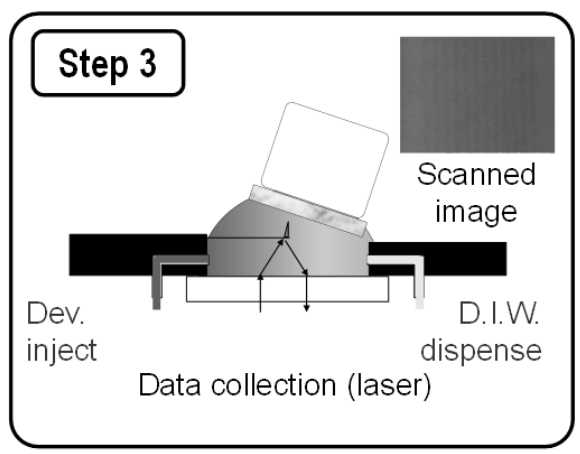

(c)

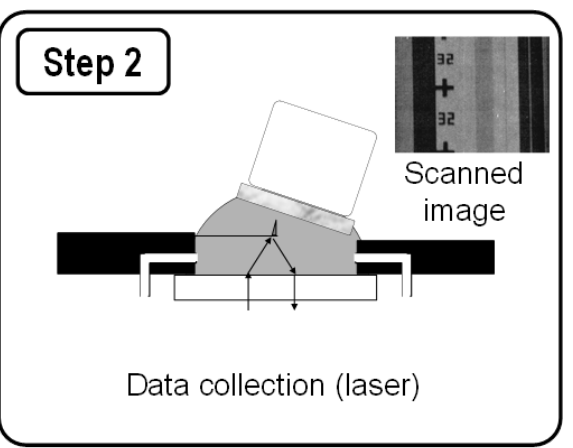

(b)

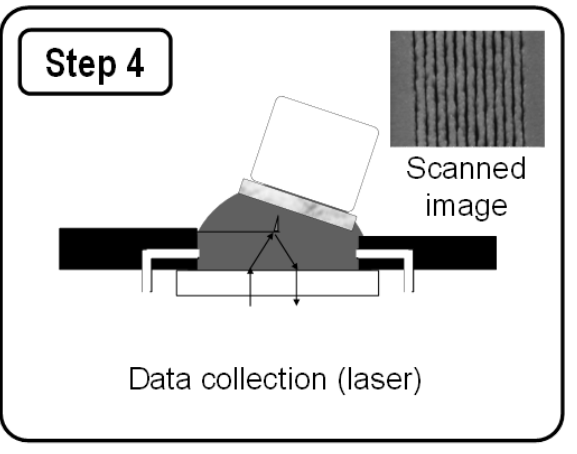

(d)

Fig. 2 Procedure utilized for the in situ resist dissolution analysis. 
volume of de-ionized water (D.I.W) was injected onto the cantilever assembly through an auto reflux system. Then, high-speed scanning was performed on the $32 \mathrm{~nm} \mathrm{hp} \mathrm{L/S} \mathrm{pattern} \mathrm{in} \mathrm{D.I.W} \mathrm{(Fig.} \mathrm{2(b)).}$ The result shows the resist film state before development. A pre-determined volume of the developer solution was injected into the developer puddle while the D.I.W. was dispensed at an optimized and constant rate through the auto reflux system (Fig. 2(c)). Finally, during the injection of the developer solution, the process of resist dissolution was recorded via continuous AFM scanning (Fig. 2(d)). For these experiments, images were obtained at an optimized HS-AFM scan rate of 0.5 frames $\cdot \mathrm{s}^{-1}$. Image scanning was performed over an area of $1000 \mathrm{~nm} \times 750 \mathrm{~nm}$ (or $400 \times 300$ pixels). The CNF cantilever utilized has a radius of curvature of $<10 \mathrm{~nm}$. All HS-AFM measurements were performed in a controlled environment inside a class 1 clean room.

\section{Results and Discussions}

\subsection{Present limitations and possible solutions}

Figure 3 shows the dissolution characteristics

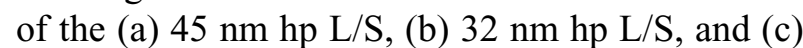
$26 \mathrm{~nm} \mathrm{hp} \mathrm{L/S} \mathrm{patterns} \mathrm{exposed} \mathrm{on} \mathrm{the} \mathrm{EUV} \mathrm{hybrid}$ model resist at the start, mid and end stages of dissolution. It can be observed from these results that dissolution characteristics (in this case; swelling of EUV exposed areas) remain the same, regardless of pattern size. From these results it is also obvious that at (c) $26 \mathrm{~nm} \mathrm{hp} \mathrm{L/S}$, the quality of scanned image has deteriorated. This was especially observed after the swelling sections have dissolved and the trenches between the lines start to get revealed. Figure 4 shows the cross section analysis results of the (a) $45 \mathrm{~nm} \mathrm{hp} \mathrm{L/S,} \mathrm{(b)}$ $32 \mathrm{~nm} \mathrm{hp} \mathrm{L/S}$, and (c) $26 \mathrm{~nm} \mathrm{hp} \mathrm{L/S} \mathrm{patterns} \mathrm{at} \mathrm{the}$ start, mid and end stages of dissolution. In this Figure, the z-coordinate or film thickness has been emphasized relative to the $\mathrm{x}$-coordinate to provide a clear comparison the resist film behavior during pattern formation. The bold lines indicate the average of a total of 11 scanned lines, as measured at the image center. The transparent boxes shown on the graph indicate the line patterns on the EUV-exposed resist film. These results clearly

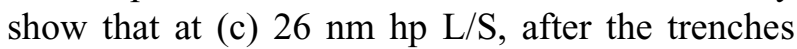
have been dissolved, scanning does not even reach half-way into the resist film.

This was expected given the comparatively thick radius of curvature of the CNF cantilever tip which is roughly $10 \mathrm{~nm}$. This can be remedied with the development of narrower cantilever tips,

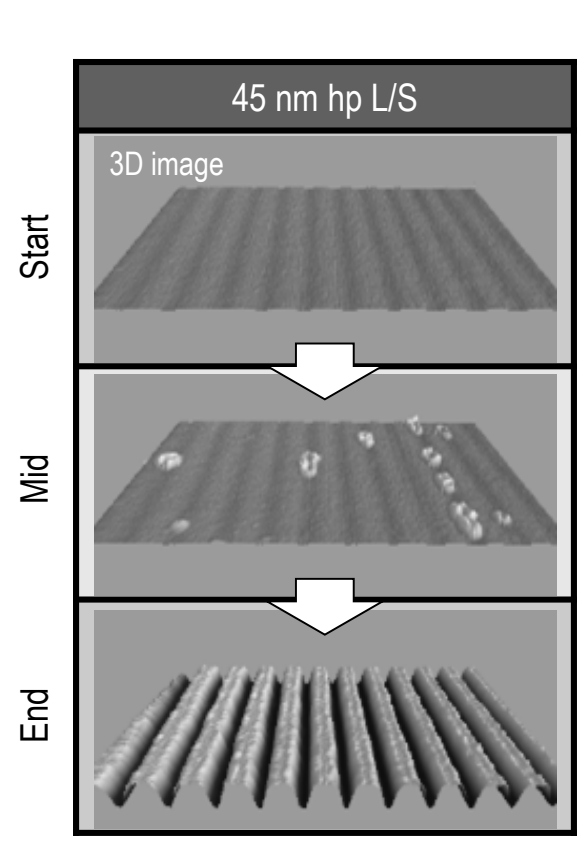

(a)

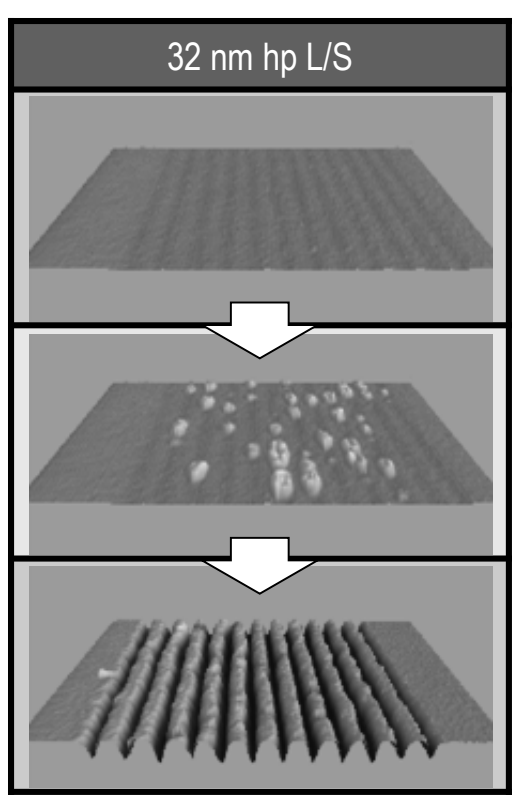

(b)

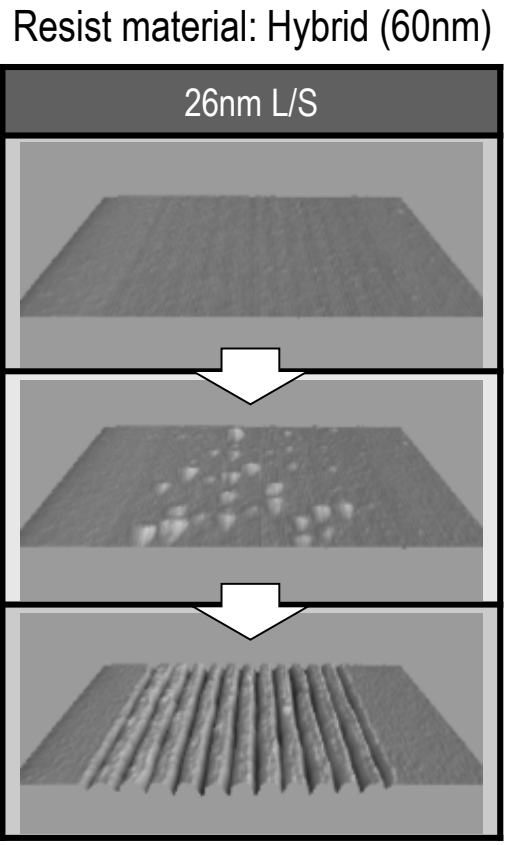

(c)

Fig. 3 The dissolution characteristics of the (a) $45 \mathrm{~nm} \mathrm{hp} \mathrm{L/S,} \mathrm{(b)} 32 \mathrm{~nm} \mathrm{hp} \mathrm{L/S,} \mathrm{and} \mathrm{(c)} 26 \mathrm{~nm} \mathrm{hp} \mathrm{L/S} \mathrm{patterns}$ exposed on the EUV hybrid model resist at the start, mid and end stages of dissolution. 


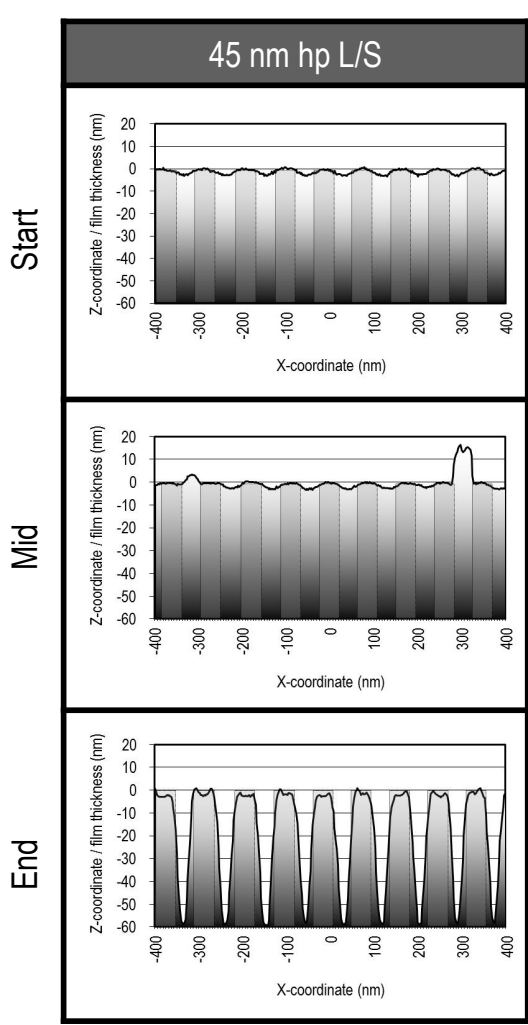

(a)

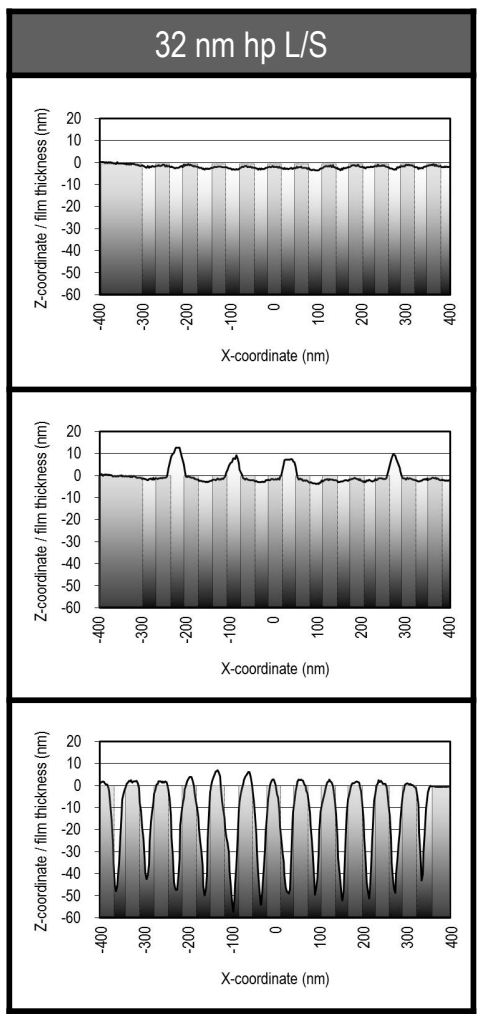

(b)
Resist material: Hybrid (60nm)

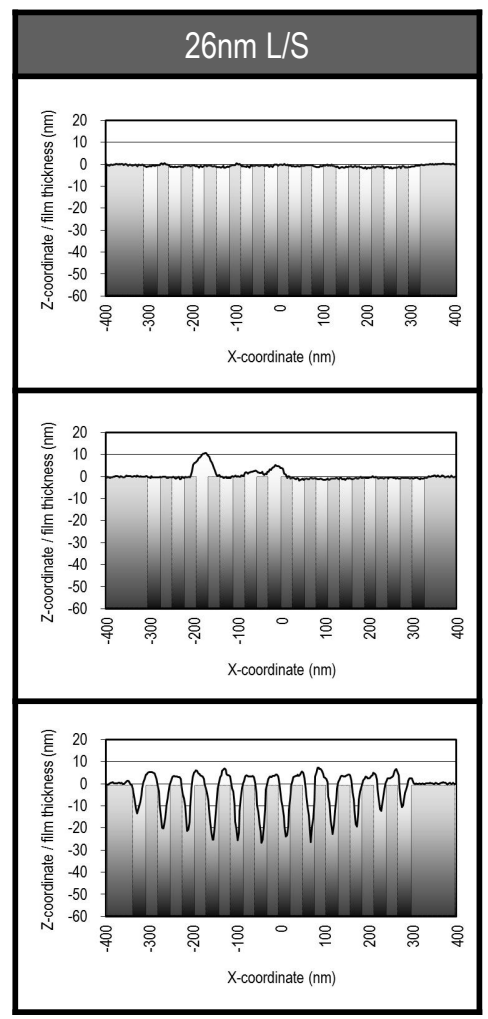

(c)

Fig. 4 The cross section analysis results of the (a) $45 \mathrm{~nm} \mathrm{hp} \mathrm{L/S,} \mathrm{(b)} 32 \mathrm{~nm} \mathrm{hp} \mathrm{L/S}$, and (c) $26 \mathrm{~nm} \mathrm{hp} \mathrm{L/S}$ patterns at the start, mid and end stages of dissolution.

preferably less than half of the presently available ones. Being able to observe the dissolution characteristics of narrower patterns is viewed important as there have been hints of how the dissolution speed varies depending on pattern size. Evaluations are currently on going on the design and application of ultra-narrow cantilevers. When this is achieved, detailed investigations are being planned on understanding the time differences between the development of these very narrow pattern features.

\subsection{Resist dissolution and line width roughness}

Figure 5 shows a comparison between the ESR1 and Resist A in terms of (a) dissolution characteristics and (b) LWR at $32 \mathrm{~nm}$ hp L/S (SEM images). As shown in the Fig. 5(b) for both resist samples, Resist A exhibits comparatively low LWR at this pattern size.

In comparison, as shown in Fig. 5(a), both resists show a certain amount of resist swelling during the first few seconds of dissolution ( $t 0)$. As dissolution advances ( $t 1$ to $t 3$ ), it can be observed that the pattern starts to show. These results show a visually appreciable difference in the quality of lines being produced between both resist samples. ESR1 shows a more rough line pattern which smoothens to a certain level after $\mathrm{t} 2$. However this was still not comparable to the smoother patterns observed with Resist A which also further smoothens at $\mathrm{t} 3$ development time.

These results confirm that LWR starts to exist during the development process. The results also show that the level of roughness changes as development time advances. Furthermore, the visual difference in line roughness between the dissolution images in Fig. 5(a) with the SEM images Fg. 5(b) shows the possible effect of the processes following dissolution; rinse and drying.

These results emphasize the possibility of defining the formation mechanism of LWR during the development process using the HS-AFM analysis method. It also shows the possibility of defining the effect of the rinse and drying on the formation of LWR. Such analysis capability will allow a clearer definition of this lithographic factor and may provide new pointers in further reducing it. However, the authors recognize that to do this, further refinements in measurement stability of the 
Film thickness: $50 \mathrm{~nm}$

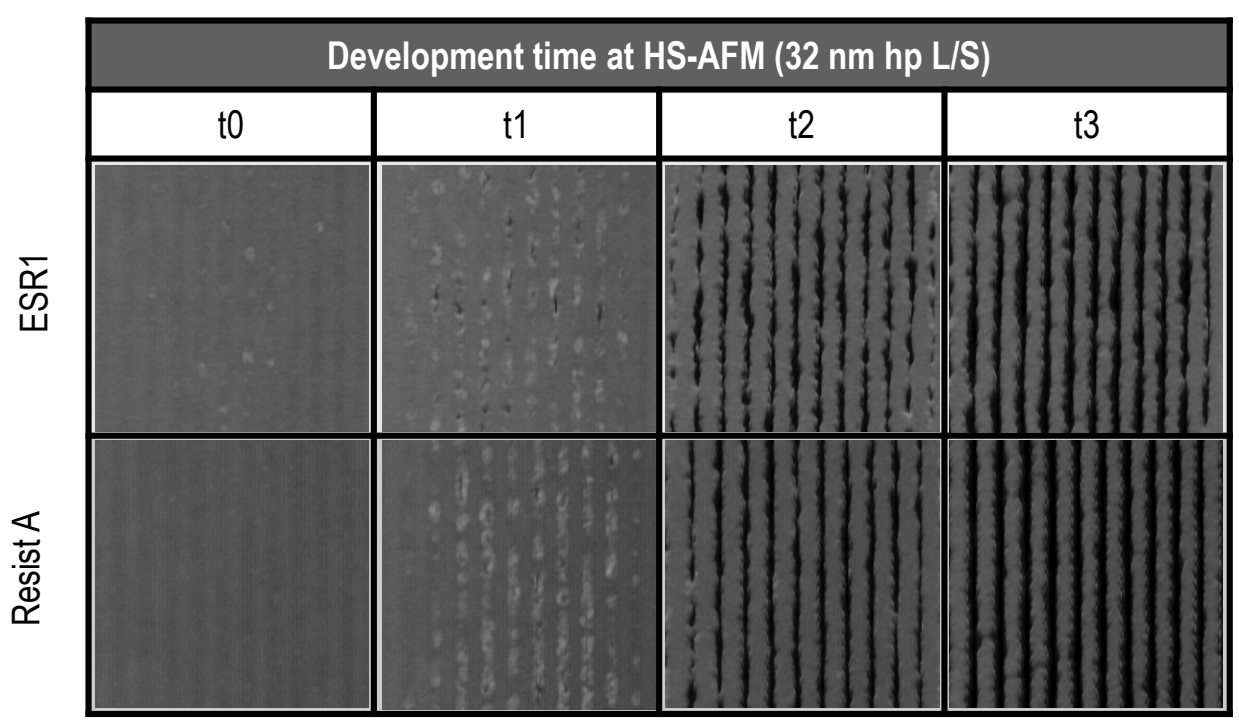

(a)

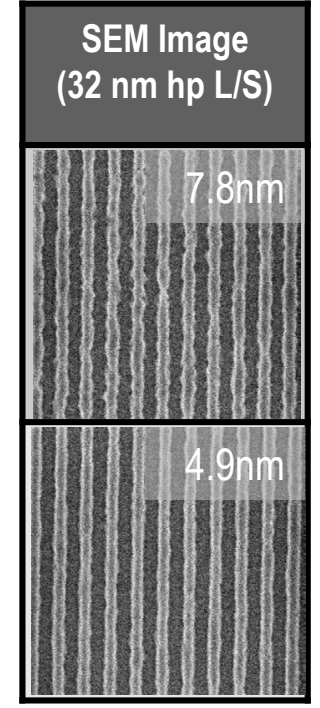

(b)

Fig. 5 A comparison between the ESR1 and Resist A in terms of

(a) dissolution characteristics and (b) LWR at $32 \mathrm{~nm} \mathrm{hp} \mathrm{L/S} \mathrm{(SEM} \mathrm{images).}$

analysis process will be necessary. Additional software in extracting LWR from these images will also play a significant role. Such technologies are presently being developed and will be reported in the near future.

\section{Summary}

The dissolution characteristics of EUV resist patterns as they are formed during the development process were analyzed using the HS-AFM. This method has been significantly optimized and can now perform the in situ dissolution analysis of 32 $\mathrm{nm} \mathrm{hp} \mathrm{L/S} \mathrm{pattern} \mathrm{developed} \mathrm{with} \mathrm{a} \mathrm{standard}$ concentration $(0.26 \mathrm{~N})$ of the TMAH developer solution. Further investigations have shown that the applicability of this method is presently limited to $\mathrm{hp} \mathrm{L} / \mathrm{S}$ patterns around $32 \mathrm{~nm} \mathrm{hp} \mathrm{L/S.} \mathrm{This} \mathrm{was}$ mainly attributed to the comparatively thick radius of curvature of presently available cantilevers. Furthermore, the results obtained here have shown that resists of good pattern LWR exhibit smooth patterns even during dissolution. It was also observed that resists of comparatively large LWR also show signs of such roughness even during the dissolution process. These results show the possibility of defining the formation mechanism of LWR during the development process.

\section{Acknowledgements}

This work was supported by the New Energy and Industrial Technology Development Organization (NEDO). We would like to thank EIDEC member companies (Advanced resist research program) for their continued support and resist and material manufacturers for providing the latest resist technologies. Lastly, we thank Dr. Motoharu Shichiri for the useful discussions and technical support on the application of the HS-AFM.

\section{References}

[1] T. Itani and T. Kozawa: Jpn. J. Appl. Phys. 52 (2013) 010002.

[2] M. Trikeriotis, M. Krysak, Y. S. Chung, C. Ouyang, B. Cardineau, R. Brainard, C. K. Ober, E. P. Gianneli, and K. Cho: Proc. SPIE 8322 (2012) $83220 \mathrm{U}$.

[3] T. Nishikubo, H. Kudo, Y. Suyama, H. Oizumi, and T. Itani: J. Photopolym. Sci. Technol. 22 (2009) 73.

[4] H. Oizumi, T. Kumise, and T. Itani: J. Photopolym. Sci. Technol. 21 (2008) 443.

[5] J. J. Santillan, K. Kaneyama, A. Morita, K. Fuse, H. Kiyama, M. Asai, and T. Itani: Proc. SPIE 8325 (2012) 832513.

[6] H. Shite, K. Matsunaga, K. Nafus, H. Kosugi, P. Foubert, J. Hermans, E. Hendrickx, M. Goethals, 
and D. Van Den Heuvel: $\quad$ Proc. SPIE 8322 (2012) 83222Y.

[7] B. Jung, C. K. Ober, M. O. Thompson, and M. Chandhok: Proc. SPIE 7972 (2011) 79722S.

[8] T. Itani and J.J. Santillan: Appl. Phys. Express 3 (2010) 096501.

[9] A. V. Pret and R. Gronheid: Microelectron. Eng. 87 (2010) 1127.

[10] T. Itani, H. Iwasaki, M. Fujimoto, and K. Kasama: Jpn. J. Appl. Phys. 33 (1994) 7005.

[11] T. Itani, H. Yoshino, S. Hashimoto, M. Yamana, N. Samoto, and K. Kasama: J. Vac. Sci. Technol. B 15 (1997) 2541.

[12] T. Itani, S. Hashimoto, M. Yamana, N. Samoto, and K. Kasama: Microelectron. Eng. 41-42 (1998) 363.

[13] T. Itani, H. Yoshino, S. Hashimoto, M. Yamana, M. Miyasaka, and H. Tanabe: J. Vac. Sci. Technol. B 16 (1998) 3726.

[14] A. Agrawal and C. L. Henderson: Proc. SPIE 5038 (2003) 1026.

[15] L. Singh, P. Ludovice, and C. Henderson: Proc. SPIE 5376 (2004) 1007.

[16] A. Kokkinis, E. S. Valamontes, and I. Raptis: J. Phys.: Conf. Ser. 10 (2005) 401.
[17] W. Hinsberg, F. Houle, S.-W. Lee, H. Ito, and K. Kanazawa: Macromolecules 38 (2005) 1882.

[18] M. Toriumi, J. J. Santillan, T. Itani, T. Kozawa, and S. Tagawa: J. Vac. Sci. Technol. B 25 (2007) 2486.

[19] C. Fonseca, B. Head, H. Shite, K. Nafus, R. Gronheid, and G. Winroth: Proc. SPIE 7969 (2011) 796911.

[20] T. Itani and J. J. Santillan: J. Vac. Sci. Technol. B 27 (2009) 2986.

[21] T. Itani and J. J. Santillan: Appl. Phys. Express 3 (2010) 061601.

[22] T. Itani and J. J. Santillan: J. Photopolym. Sci. Technol. 23 (2010) 639.

[23] T. Itani and J. J. Santillan: Proc. SPIE 7972 (2011) $79720 \mathrm{H}$.

[24] T. Itani: J. Photopolym. Sci. Technol. 24 (2011) 111.

[25] J. J. Santillan and T. Itani, Jpn. J. Appl. Phys. 51, 06FC06 (2012).

[26] J. J. Santillan and T. Itani: Proc. SPIE 8682 (2013) 86820I.

[27] T. Ando, T. Uchihashi, N. Kodera, D. Yamamoto, A. Miyagi, M. Taniguchi, and H. Yamashita: Pflugers Arch. 456 (2008) 211. 